\title{
Editorial: Structural Health Monitoring of Bridges
}

\author{
Neil A. Hoult ${ }^{1}$ and Branko Glisic ${ }^{2 *}$ \\ ${ }^{1}$ Department of Civil Engineering, Queen's University, Kingston, ON, Canada, ${ }^{2}$ Department of Civil and Environmental \\ Engineering, Princeton University, Princeton, NJ, United States
}

Keywords: structural health monitoring, bridges, data analysis, sensing technologies, real-life applications

\section{Editorial on the Research Topic}

\section{Structural Health Monitoring of Bridges}

Structural health monitoring (SHM) has been the focus of research for several decades. During that time, it has seen a number of firsts and advances that have kept it a thriving research field. However, in order for it to become a widely used tool in industry there are still a number of grand challenges to overcome. When it comes to SHM of bridges, these grand challenges include: (i) the development of cost effective and robust sensors, (ii) assessing these sensor technologies in real world settings, and (iii) identifying applications where monitoring can have an impact. Within this special issue there are papers that make a significant contribution toward addressing each of these challenges.

In terms of the development of cost effective and robust sensors, the article by Gibbs et al. introduces the reader to the notion of a "citizen sensing protocol." In this novel approach to SHM, low-cost rapidly deployable sensors are used to aid in the design of footbridges in remote regions. Bridges that would otherwise be susceptible to wind induced vibrations due to their flexibility and light weight, can now be designed appropriately using empirical equations and approaches that result directly from this SHM approach. In Aono et al., the reader is introduced to a sensing technology that provides a sensor life of 20 years. After introducing lab-based studies, the Mackinac Bridge in Michigan is used as the case study to demonstrate the potential of this sensor technology. In both papers, researchers have sought to address both the cost and robustness issues that often prevent the uptake of SHM by industry.

Several papers in this special issues also seek to illustrate the use of SHM through real world case studies. In Martín-Sanz et al., the research team illustrates the benefits of Ultra-high-performance fiber-reinforced cement-based composites (UHPFRC) by applying them to an actual bridge structure. The Buna Bridge, in Croatia, was a steel bridge built in 1893 and decommissioned in 2010. The research team was able to test the bridge both statically and dynamically before and after rehabilitation with UHPFRC. Using a suite of sensor technologies including embedded fiber optics, acoustic emission sensors, and digital image correlation they were able to assess the impact of the rehabilitation using this high compressive strength, low permeability, and high ductility material. The SHM data along with a finite element analysis were used to prove that UHPFRC could extend the lifetime of a structure. In Barrias et al., the research team from UPC-BarcelonaTech demonstrated the practical use of Distributed Optical Fiber Sensors (DOFS). They not only presented their lab-based evaluations of new implementation methods, comparison, and performance analysis of different bonding adhesives, and spatial resolution but also a case study from Barcelona highlighting the use of DFOS in the field. Here again, research projects such as these are helping to demonstrate to industry the value of SHM through case studies on real infrastructure assets.

Finally, the research community must demonstrate potential applications of SHM if there is to be widespread adoption. Paper by Kliewer and Glisic does just that by focusing on the measurement 
of displacements, which is key to the assessment of bridge structures. The article introduces five strain based displacement estimation techniques using of fiber Bragg grating (FBG) sensors. The approaches are rigorously evaluated using both analytical and numerical modeling, and the strengths and weaknesses of each approach are highlighted. The research team then demonstrates the techniques using data from an in-service bridge. In Ngeljaratan and Moustafa, an established measurement technique is demonstrated for a new application. The research team uses digital image correlation (DIC) to perform damage identification on three $1 / 3$ scale model bridges. By first demonstrating the use of DIC for system identification, they are then able to show how it can be used to estimate natural frequency and damping ratio before and after damage. In both cases, the researchers were able to demonstrate how SHM can be used to provide engineers with critical data for structural evaluation and thus assist adoption by industry.

While SHM still has obstacles to overcome before it reaches widespread use and acceptance by industry, nevertheless, this special issue demonstrates how world leading researchers are taking steps to address these grand challenges. And the quality and breadth of their work suggests that it will not be long before SHM of bridges is commonplace.

\section{AUTHOR CONTRIBUTIONS}

$\mathrm{NH}$ wrote the original draft of the editorial. BG revised and submitted it.

\section{ACKNOWLEDGMENTS}

The editors would like to thank to all contributors to this special issue as well as the reviewers and editorial team of Frontiers.

Conflict of Interest: The authors declare that the research was conducted in the absence of any commercial or financial relationships that could be construed as a potential conflict of interest.

Copyright (c) 2020 Hoult and Glisic. This is an open-access article distributed under the terms of the Creative Commons Attribution License (CC BY). The use, distribution or reproduction in other forums is permitted, provided the original author(s) and the copyright owner(s) are credited and that the original publication in this journal is cited, in accordance with accepted academic practice. No use, distribution or reproduction is permitted which does not comply with these terms. 